\title{
Optimization Based Liver Contour Extraction of Abdominal CT Images
}

\author{
Jayanthi Muthuswamy ${ }^{1}$, B. Kanmani ${ }^{2}$ \\ ${ }^{1}$ Department of Electronics and Communication Engineering, BMS College of Engineering, India \\ ${ }^{2}$ Department of Telecommunication, BMS College of Engineering, India
}

\begin{tabular}{l} 
Article Info \\
\hline Article history: \\
Received Mar 5, 2018 \\
Revised Jul 15, 2018 \\
Accepted Jul 22, 2018 \\
\hline
\end{tabular}

Keyword:

Bilateral filter

Fuzzy C means

Label Connected Component

Optimization

Preprocessing

\begin{abstract}
This paper introduces computer aided analysis system for diagnosis of liver abnormality in abdominal CT images. Segmenting the liver and visualizing the region of interest is a most challenging task in the field of cancer imaging, due to small observable changes between healthy and unhealthy liver. In this paper, hybrid approach for automatic extraction of liver contour is proposed. To obtain optimal threshold, the proposed work integrates segmentation method with optimization technique in order to provide better accuracy. This method uses bilateral filter for preprocessing and Fuzzy C means clustering (FCM) for segmentation. Mean Grey Wolf Optimization technique (mGWO) has been used to get the optimal threshold. This threshold is used for segmenting the region of interest. From the segmented output, largest connected region is identified using Label Connected Component (LCC) algorithm. The effectiveness of proposed method is quantitatively evaluated by comparing with ground truth obtained from radiologists. The performance criteria like dice coefficient, true positive error and misclassification rate are taken for evaluation.
\end{abstract}

Copyright $\odot$ 2018Institute of Advanced Engineering and Science. All rights reserved.

\section{Corresponding Author:}

Jayanthi Muthuswamy,

Research Scholar,

Department of Electronics and Communication Engineering,

BMS College of Engineering, Bangalore, India.

Email: jayanthi.sathishkumar@live.com

\section{INTRODUCTION}

Liver is the largest and most important organ for survival. It is one and a half $\mathrm{kg}$ organ and located in the upper right quadrant of the abdominal cavity [1]. The liver performs important functions like filter the blood, process the fats and to metabolize and store carbohydrates. It is also prone to several distinct types of liver ailments. Liver diseases have different colors such as blue indicate cyst, yellow indicate fatty liver, brown is fibrosis etc. Different imaging techniques like ultrasound, computed Tomography Imaging, Magnetic resonance imaging, positrons emission tomography etc are available for diagnosis of liver diseases [2].

Among these, CT scan is a well known non-invasive imaging modalities and it is more preferred by diagnosticians since they have high signal to noise ratio, good spatial resolution, patient friendly protocols, less cost and less examination time. It also provides more accurate anatomical information about the visualized structure. Manual segmentation of liver is tedious and time consuming task for radiologists [3]. Automatic segmentation is more troublesome because of low contrast, multiple slices of CT image and similar intensity of both the liver and tumor. Thus, there is a need for Computer aided analysis system to diagnose the liver abnormality from the huge amount of medical data.

The main goal of computer aided analysis system is to provide computer output, as a second opinion to assist physician in the detection of abnormalities and to improve the segmentation accuracy. This system 
also reduces the image reading time. This system has various phases: Data acquisition, preprocessing, combined segmentation and optimization algorithm and post processing. Clustering is one of the most popular segmentation techniques used to organize the objects into groups. The Fuzzy C-Means algorithm is a clustering algorithm where each item may belong to more than one group (hence the word 'fuzzy'), where the degree of membership for each item is given by a probability distribution of the clusters.

However, it suffers from the possibility of falling into local minima. To overcome this drawback, the FCM algorithm is combined with another soft computing algorithm. Grey wolf optimization technique is a new meta-heuristic optimization algorithm based on the social hierarchy and hunting behavior of grey wolves and it is used to search and hunt a prey (solution) [4]. This technique is used to optimize the cluster center in order to find the optimal threshold. This technique is used in the proposed work. The rest of this paper is organized as follows: Section 2 gives a detail description of the proposed method. Section 3 presents experimental results and performance analysis. Section 4 provides the conclusions and future scope.

Liver segmentation still remains an open challenge problem for researchers. Many researchers have developed different methods and techniques to extract the liver and tumor from the abdominal CT images over the recent years. And number of researches has been carried out on soft computing based optimization methods. This literature survey has been done on three phases: preprocessing, segmentation and optimization. The existing methods used for the extraction of liver contour are discussed in this section.

In Russo and Sonali author presented linear filtering method to remove Gaussian noises in a CT images. The main drawback in their work is blurring problem [5, 6]. To overcome this, Chaux et al have developed nonlinear filter [7]. This filter not only removes the blurring effect and also preserves the edge information; thereby improve the effectiveness of non-linear filter. Proposed bilateral filter to preserve the edges and image smoothening using nonlinear combination of image pixels [8]. Therefore, it can be seen that noisy CT image will degrade the quality of an image so preprocessing is essential in computer aided analysis system.

In S. Gunasundari and M. Suganya author has reviewed comparative study of liver segmentation methods to extract the liver contour and also discussed the limitation of various methods [9]. K Mala et al have proposed adaptive threshold based morphological processing for liver segmentation [10]. M Jayanthi et al have proposed an approach for extracting the liver and tumor from abdominal CT images and used for computer aided diagnosis [11]. The authorshave used seeded region growing method and focused on limited amount of sample image and performance measures not evaluated in their work. SS Kumar et al have developed a CAD system for segmenting the liver and tumor extraction [12].

Ahmed Fouad Ali et al have presented a novel approach based on nature inspired optimization algorithms and highlighted the problem present in the CT liver segmentation [13]. The authors have explained how the nature inspired algorithms can be applied to solve the segmentation problem. Gehed Ismail et al have presented a Computer aided diagnosis system for abdominal CT images [14]. The authors have proposed a hybrid method to reduce the false positive error rate. In Mittal $\mathrm{N}$ et al the authors have extended a modified mean grey wolf optimization approach for biomedical problems and the performance was compared with other meta-heuristic optimization algorithms [15].

Therefore, it can be seen that there are different methods to extract the liver contour. Some of the methods discussed in the literature survey used prior knowledge of region of interest and a single segmentation method for segmenting the liver contour from abdominal CT images. Sometimes, these methods might create fake segmentation error. In Edy Fradinata et al and Ratna Nit in Patil et al the authors have explained the importance of optimization and evaluation of classification algorithms [16, 17]. The next section discusses about the limitation of existing methods and also explains how the proposed method is used for liver segmentation in computer aided analysis system.

\subsection{Research Problem}

The significance of liver segmentation problems are as follows

a. Automatic segmentation of liver is a difficult task due to the overlapping of region of liver with adjacent organs and the intensity of liver is same as that of other organs.

b. Existing methods used pixel-based distribution for finding the liver contour.

c. Most of the researcher focused on single segmentation method, but this method incapable to solve the complex problem like liver shape variation among the patients.

d. Machine learning based liver segmentation method finds laboriously to calculate optimal threshold.

So the proposed work integrates machine learning with optimization method to improve the efficacy of segmentation results and also provide the optimal solution of finding the cluster centers. Therefore, the problem statement of the proposed work can be stated as "To develop a precise segmentation method that incorporate FCM with bio inspired optimization method for the extraction of liver contour from the abdominal CT images". 


\subsection{Proposed Solution}

The proposed solution is an extension of our hybrid method towards liver diagnosis problem [18]. We highlighted the importance of hybrid techniques for the extraction of liver contour. Therefore, in this proposed solution, we emphasize bio inspired optimization method in order to find the optimal threshold that can be used by radiologists for the extraction of liver contour is discussed. The proposed system has various phases to obtain the region of interest. Figure 1 shows the various phases involved in the proposed system.

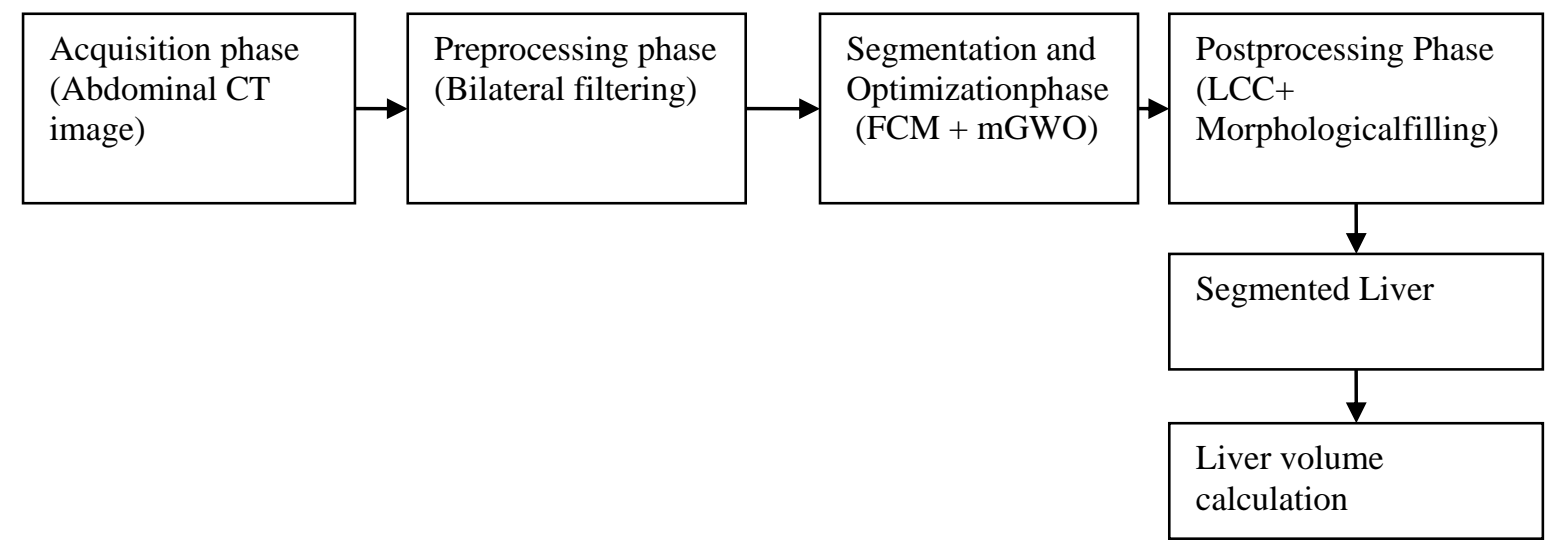

Figure 1. Block diagram of the proposed system

\section{PROPOSED METHOD IMPLEMENTATION}

The various steps involved in the extraction of liver contour are discussed in this section. The primary step in computer aided analysis system is a collection of image database for abdominal CT images. Then, preprocessing filter is discussed in order to improve the quality of an image. Followed by this, FCM based optimization and label connected algorithms are explained to get the liver contour of abdominal CT images. The various phases in the proposed method are clearly explained in the accompanying passage.

\subsection{Abdominal CT Image}

Computed Tomography scan combines series of X-ray images that are taken from different angles, and uses computer processing to produce cross sectional images of the human body. It provides the detail information of the internal organs and also helps radiologists to diagnose the diseases. For a patient, more than 150 slices of CT image are obtained. A suitable selection of CT Image is essential. Middle slice is preferable and gives more detailed information. Both real time database and simulated database are used in the proposed work. Our database is a collection of various images of CT abdominal organs which include normal, fatty and over extended liver with tumor.

\subsection{Bilateral Filter}

In order to improve the quality of a CT image, preprocessing is essential. Bilateral filter is used as preprocessing filter. It is non-linear, non-iterative, edge preserving smoothing filter developed by Tomasi [19]. The main noise in the CT image is Gaussian noise. Bilateral filter is obtained by the combination of weighted function of two Gaussian filters: partial domain and intensity domain. The intensity value of each pixel is replaced by weighted average. Let $G$ is an image. Then $G p$ is the value of the image $G$ at pixel position $\mathrm{u}$. Bf $(\mathrm{G})$ is the output of bilateral filter applied to an Image $\mathrm{G}$

Spatial distance is calculated as

$$
s_{p d}(u, v)=e^{-\|u-v\|^{2}} / 2 \sigma_{s d}^{2}
$$

intensity difference is calculated as

$$
I_{i d}(p, q)=e^{-|U(u)-U(v)|^{2}} / 2 \sigma_{i d}^{2}
$$

guassian kernel coefficient are $\sigma_{\mathrm{sd}}$ and $\sigma_{\mathrm{id}}$ which controls spatial distance and intensity difference. These coeffieients are directly proportional to image size and edge amplitude and these are mainly used to control the weights in spatial and intensity domain. At pixel $\mathrm{p}$ 


$$
\operatorname{Bif}(p)=\frac{1}{C} \sum_{q \in \gamma(p)} \operatorname{Bif}(q) s_{p d}(p, q) I_{i d}(p, q)
$$

where $\mathrm{C}$ is a normalization factor $c=\sum S_{p d}(u, v) I_{i d}(u, v)$ and $\gamma(p)$ shows the spatial neighborhood of $\operatorname{Bif}(p)$. Each pixel is replaced by the weighted average of nearby pixels in spatial neighborhood. Shows the spatial neighborhood of $\mathrm{Bf}(\mathrm{p})$. The pseudo code of bilateral filter is shown in Table 1 .

Table 1. Pseudo Code for Bilateral filter

\begin{tabular}{l} 
Input: CT Image ; Output: Preprocessed image \\
\hline 1. DICOM image is converted to JPEG using acculite software. \\
2. Convert that CT image to grey scale image. \\
3. Define the bilateral filter parameter w, osd and $\sigma$ id \\
4. For all the pixel, do the following steps 5,6,7 \\
5. Calculate the spatial distance using the equation 1 \\
6. Calculate the intensity difference using the equation 2 \\
7. Apply the filtering values on CT grey scale image using the equation 3. \\
8. Get the resultant pre processed image. \\
\hline
\end{tabular}

\subsection{Fuzzy C Means Algorithm}

FCM [20] based objective function is a most popular iterative clustering algorithm that allows the most precise formulation of the clustering criteria. The basic concept is to find optimal cluster center that minimize objective function. The basic concept is to partition a data set $\mathrm{DX}=\left\{\mathrm{DX}_{1}, \mathrm{DX}_{2} \ldots . \mathrm{DX}_{\mathrm{M}}\right\}$ into ' $\mathrm{C}$ ' number of clusters. First step in FCM is to calculate the degree of membership function. For a given data point $\mathrm{DX}_{\mathrm{i}}$, the degree of its membership to cluster $\mathrm{j}$ is calculated as follows;

$$
U M_{i j}=\frac{1}{\sum_{K=1}^{C}\left(\frac{\left\|D X_{i}-C_{j}\right\|}{\left\|D X_{i}-C_{K}\right\|}\right)^{\frac{2}{m-1}}}
$$

where $\mathrm{m}$ is a fuzziness coefficient, value of $\mathrm{i}$ ranges from 1 to $\mathrm{M}$ and $\mathrm{j}$ varies from 1 to $\mathrm{C}$. Then calculate cluster centroid based on weighted average using equation

$$
C_{j}=\frac{\sum_{i=1}^{M} U M_{i j}^{m} D X_{i}}{\sum_{i=1}^{M} U M_{i j}^{m}}
$$

next, calculate Euclidean distance between cluster vectors to the data point using the formula

$$
E u c_{-} D=\left|D X_{i}-C_{j}\right|^{2}
$$

this distance finds the closeness of each data point to the cluster vector $\mathrm{Cj}$. For every iteration of FCM algorithm, the following objective point is minimized.

$$
O b J_{m}=\sum_{i=1}^{M} \sum_{j=1}^{C} U_{i j}\left|D X_{i}-C_{j}\right|^{2}
$$

if $\left(\mathrm{ObJ}_{\mathrm{m}}-\mathrm{ObJ}_{\mathrm{m}-1}\right)<=\varepsilon$ is satisfied, following iteration is terminated. The value of $\varepsilon$ ranges from 0 to 1 .

\subsection{Grey Wolf Optimization}

Mirijali et al proposed a new heuristic optimization algorithm. This algorithm mimics social hierarchy and hunting behavior of grey wolves in nature [21]. The advantage of grey wolf is simple, easily be programmed and does not need specific input parameters. Generally, Grey wolves live in a group (pack), each group has an average member of 5-10. All the members in the group have a own power of dominant hierarchy as shown in Figure 2(a). Four types of grey wolves alpha $(\alpha)$, beta $(\beta)$, delta $(\delta)$, and omega $(\omega)$ are worked for simulating the leadership hierarchy. This is used to search and hunt the prey (solution) and the leadership priority level to plan the hunting are $\alpha, \beta, \delta$ and $\omega$ respectively. The wolves omega $\omega$, and are responsible for guiding the search (hunting), while other wolves follow. Three steps of the hunting behavior are encircling, hunting and attacking the prey. 


\section{Encircling the prey}

When the grey wolves start hunting, they encircle prey. The encircling behavior is presented as a mathematical model and given as

$$
\begin{aligned}
& D=\left|C \cdot X_{\text {pre }}(t)-X_{g p}(t)\right| \\
& n X(t+1)=X_{\text {pre }}(t)-A . D \\
& A=2 a r_{1}-a \\
& C=2 r_{2}
\end{aligned}
$$

Where $\mathrm{t}$ is a current iteration, $\mathrm{X}_{\mathrm{pre}}$ is a prey position vector, $\mathrm{X}_{\mathrm{gp}}$ is the grey wolf vector position and $\mathrm{A}, \mathrm{C}$ are the coefficient vector. Random vector is $\mathrm{r} 1$ and $\mathrm{r} 2$, the values in the range of $[0,1]$.

The alpha guides the hunting process and beta and delta might have a part in hunting. The updating position of grey wolves is shown in Figure 2(b). The location of prey position is expected to come from the alpha, beta, and deltawolves $\mathrm{q}$ and given by the following equation

$$
\begin{aligned}
& D_{\alpha n}=\left|C_{1} \cdot X_{\alpha}(t)-X_{g p}(t)\right| \\
& D_{\beta n}=\left|C_{2} \cdot X_{\beta}(t)-X_{g p}(t)\right| \\
& D_{\delta n}=\left|C_{3} \cdot X_{\delta}(t)-X_{g p}(t)\right| \\
& X_{1 f}=X_{\alpha}(t)-A_{1} D_{\alpha n} \\
& X_{2 f}=X_{\beta}(t)-A_{2} D_{\beta n} \\
& X_{3 f}=X_{\delta}(t)-A_{3} D_{\delta n} \\
& X(t+1)=\frac{X_{1 f}+X_{2 f}+X_{3 f}}{3}
\end{aligned}
$$

\section{Attacking}

When the prey stops moving, the grey wolves end the hunting process. This process is mathematically expressed by decreasing $a$ from 2 to 0 . If $|A v|<1$, wolves move towards the prey for attacking. The pictorial representation of grey wolves updating position is shown in Figure 2(b).

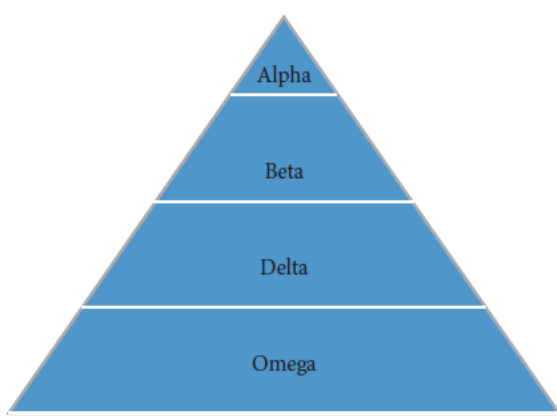

Figure 2(a). Social hierarchy of wolves [22]

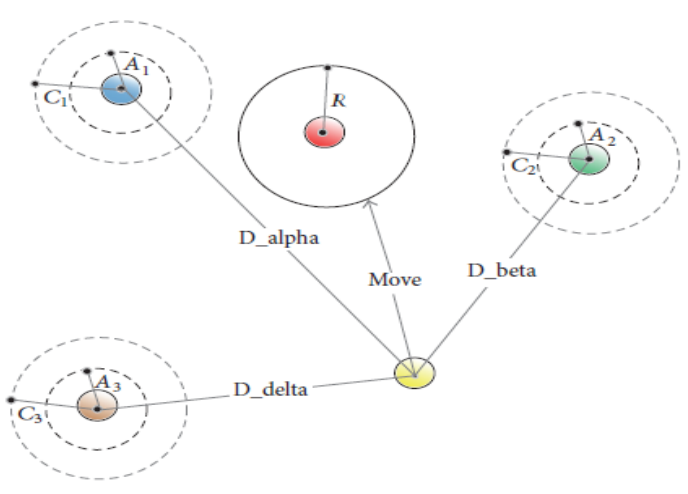

Figure 2(b). Position updating of grey wolf [22]

in m-GWO optimization algorithm, mean is taken into consideration. This algorithm is used to find the optimal threshold values in order to improve the clustering results produced by FCM. The Psuedo code for $\mathrm{m}-\mathrm{GWO}$ optimization Algorithm is shown in Table 2. 
Table 2. m-GWO algorithm pseudo code

1. Read the enhanced bilateral filter output

2. Get cluster center $\mathrm{Cj}$ where $\mathrm{j}=1,2, \ldots$ num of cluster using equation 4 .

3. Initialise m-GWO parameters - search agents, dimension, maximum iteration, lower and upper search boundary.

4. Generate wolves position Pi randomly on size of the pack.

5. Assign $\mathrm{Pj}=\mathrm{Cj}$ that is jth pack of ith wolves is $\mathrm{Cj}$.

6. Initialize alpha, beta , delta.

7. Initialize wolves positions $\mathrm{P} \alpha, \mathrm{P} \beta, \mathrm{P} \delta$

8. Set $1=0($ iteration)

9. While $1<\max$ it do

10. for each search agent do

11. Calculate the fitness of each search agent.

12. Upadate current search agent position based on fitness.

13. End for

14. Update $\mathrm{P} \alpha, \mathrm{P} \beta, \mathrm{P} \delta$ using equation 9-15 (by inserting mean before $\mathbf{X}$ )

15. Set $1=1+1$

16. End while

17. Use the best solution to generate the partition matrix Uij .And generate the final cluster with the partition matrix.

\subsection{Label Connected Algorithm}

The algorithm has two modules: (i) Labeling of connected components (ii) Search for the largest component [23]. For the output of label connected component, apply morphological opening filter is used to fill the holes. The superposition of the contour on the original image allows us to deduct the region of the liver. To obtain the liver region, liver mask is multiplied with original image.

\section{RESULTS AND ANALYSIS}

The proposed approach is analyzed, applied and tested on abdominal CT image dataset [24]. In this work, 20 Images is taken for analysis. All images are axial images. The proposed works are carried out on Pentium processor and implemented in MATLAB 9. The parameter setting of bilateral filter and $\mathrm{m}-\mathrm{GWO}$ is shown in Table 3.

Table 3. Parameter setting

\begin{tabular}{|c|c|c|}
\hline Term & Description & Values \\
\hline $\mathrm{w}$ & Window size & 5 \\
\hline osd & Spatial distance & 3 \\
\hline oid & Intensity difference & 0.1 \\
\hline num_clus & Number of fuzzy clusters & 3 \\
\hline $\mathrm{m}$ & Fuzziness & 2 \\
\hline no_search & Number of search agents & 20 \\
\hline Max_it & Number of iteration & 10 \\
\hline lb,ub & Lower and upper bound & {$\left[\begin{array}{ll}0 & 255\end{array}\right]$} \\
\hline
\end{tabular}

The abdominal CT image is converted to gray scale image. To remove the noise artifacts, preprocessing is applied on gray level image CT images. Input to the bilateral filter is normalized with closed interval of $[0,1]$. Since image was normalized, parameter of bilateral filter was also normalized by dividing with maximum intensity. The output of bilateral filter is shown in the Figure 3.
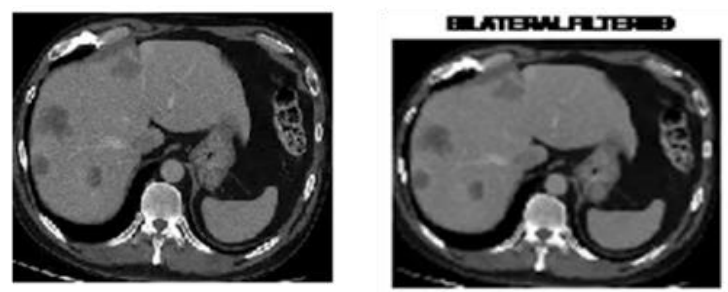

Figure 3. Gray scale CT image and output of bilateral filter 
The output of bilateral filter is taken for next phase. With the help of mGWO optimization method, three cluster centers of optimized values were obtained. These values were used by FCM algorithm. The corresponding cluster output is shown in Figure 4.
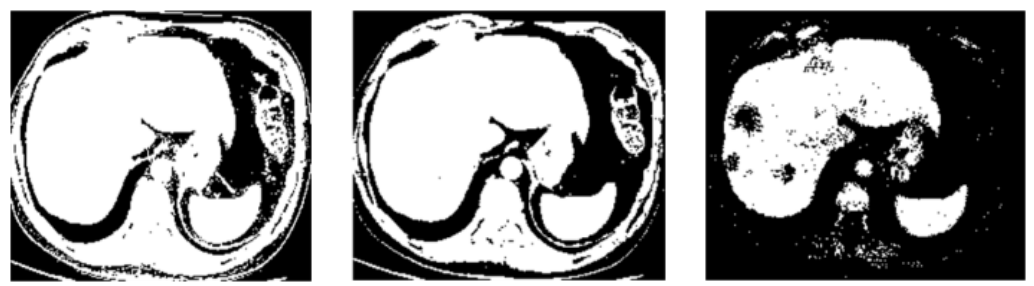

Figure 4. FCM output of different clusters

The best cluster was taken for post processing. In post processing phase, largest connected region was identified and morphological filling was done. This gives segmented liver contour as shown in Figure 5.
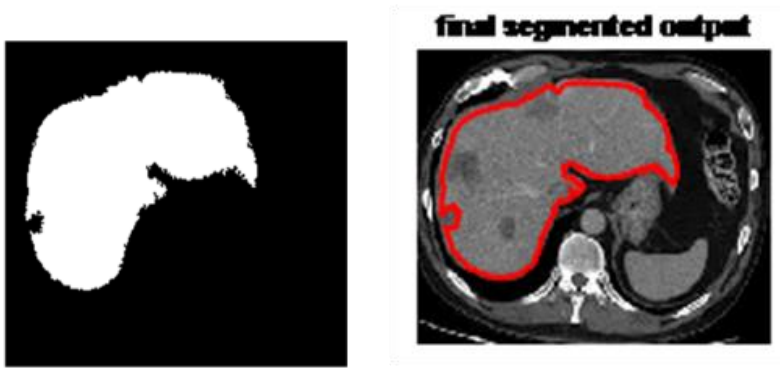

Figure 5. Liver contour output and superimposed liver output with original image

After obtaining the segmented liver, contour detection is used for the visualization. The volume of the liver is calculated using the formula given below. For sample database,

Volume of liver $=$ slice thickness* Area

Area $=$ Number of pixels $*$ Pixel dimension

The experimental results of proposed method are compared with other segmentation methods that were discussed in [25].The output of all methods is painted in Figure 6. From the figure it is inferred that FCM based optimization method gives better results. But the eye of the mind indicates that optimization results are admirable when compared to all other methods. This conclusion is purely based on visualization. To understand thebest segmentation method, following performance measures are done.

Histogram based liver segmentation

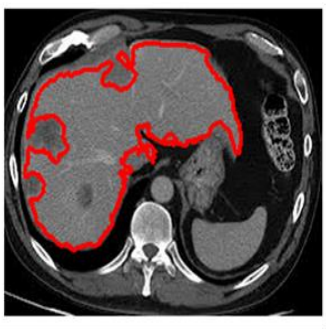

Seeded region growing based liver segmentation
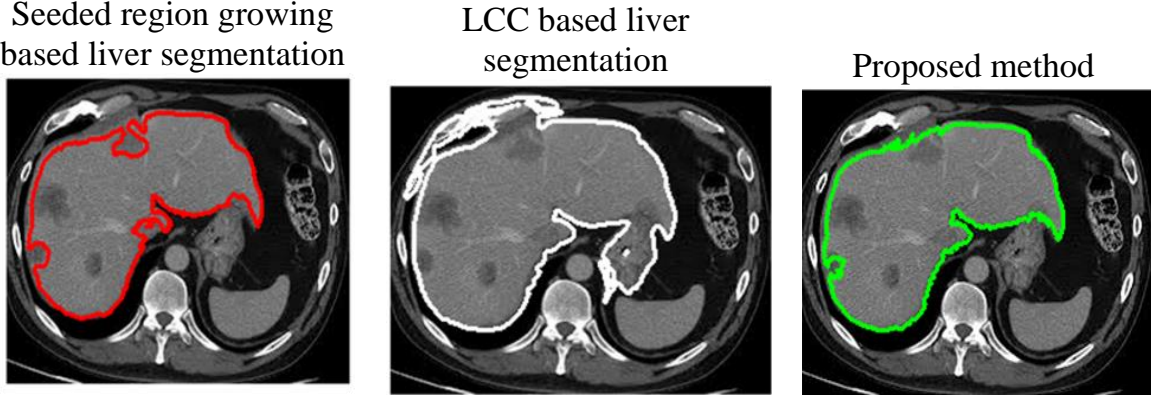

Figure 6. Results of various segmentation methods 
Dice coefficient gives the similarity between automatic segmentation and ground truth image. And it defined as follows

$$
\text { Dice }=\frac{2|\mathrm{~A} \cap \mathrm{M}|}{|\mathrm{A}+\mathrm{M}|}
$$

True positive fractionalso called Sensitivity and Recall, measures the portion of positive vowels in the ground truth that are also identified as positive by the segmentation being evaluated.

$$
\begin{aligned}
& \text { True Positive fraction }=\frac{|\mathrm{A} \cap \mathrm{M}|}{\mathrm{M}} \\
& \text { Misclassification Rate }=1-\frac{|\mathrm{A} \cap \mathrm{M}|}{\mathrm{M}}
\end{aligned}
$$

$\mathrm{A}$ is number of pixels of the automatically segmented liver regions and $\mathrm{M}$ is number of pixels of the manually segmented liver (ground truth) by the experts. Above metrics are used to find the similarity between automatic and manual segmented output (GT). Table 4 shows the statistical analysis of segmented output. Proposed method (PM) runs for Dataset contains 20 images and this table illustrates the detailed outcome of proposed work in terms of the dice coefficient, true positive rate and misclassification rate. The accuracy is more if the misclassification rate is less.

Table 4. Performance metrics of proposed method

\begin{tabular}{llrrrr}
\hline Dataset & PM & GT & TPF & DICE & MCR \\
\hline ID1 & 20054 & 22123 & 0.904 & 0.9217 & 0.096 \\
ID2 & 2788 & 3103 & 0.8984 & 0.9023 & 0.1016 \\
ID3 & 6612 & 7258 & 0.9109 & 0.9318 & 0.0891 \\
ID4 & 10312 & 11886 & 0.892 & 0.9132 & 0.108 \\
ID5 & 1852 & 1942 & 0.9536 & 0.9264 & 0.0464 \\
\hline
\end{tabular}

The performance of proposed work is compared with [25] and the results are tabulated in Table 5, it can be observed that accuracy of the proposed work is improved. Thus, FCM based optimization method performed well for extracting the liver contour and it can be used in computer aided analysis system to find the abnormality in the liver contour.The dice coefficient plot are represented in Figure 7.

Table 5. Comparison of other segmentation techniques in terms of dice coefficient

\begin{tabular}{ccccc}
\hline Methods & DT2 & DT3 & DT4 & DT5 \\
\hline Histogram & 0.67 & 0.652 & 0.689 & 0.7312 \\
SRG & 0.78 & 0.754 & 0.734 & 0.7862 \\
LCC & 0.83 & 0.841 & 0.841 & 0.891 \\
Proposed & 0.90 & 0.9318 & 0.913 & 0.9264 \\
\hline
\end{tabular}

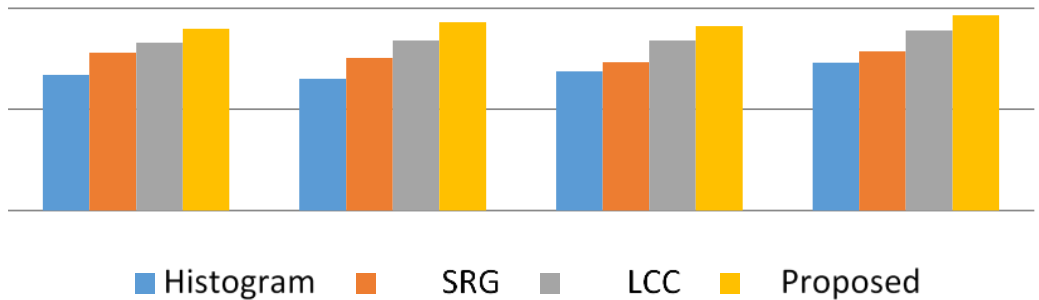

Figure 7. Dice coefficient plot of various segmentation methods 


\section{CONCLUSION}

The Proposed algorithm uses hybrid method, combination of FCM and mean Grey wolf optimization algorithm with label connected component algorithm for liver segmentation. The performance of the proposed method was compared with existing methods and the proposed method achieved the accuracy of $91 \%$ for liver segmentation. The proposed work is mainly used to identify the suspicious pixels from the liver region and helps radiologists for diagnosing liver diseases. For the future studies, other optimization algorithms will be compared with proposed method for different medical imaging problems.

\section{REFERENCES}

[1] Richard Carlton, Arlene Mckenna Adler, "An overview of imaging modalities principles of radiographic imaging: An art and A science", cengagelearning, $5^{\text {th }}$ edition, 2012.

[2] Akshat Gotra, Lojan Sivakumaran, "Liver segmentation: indications, techniques and future directions", Insight imaging, vol 8, pp 377-392, 2017.

[3] Jayanthi, M., Kanmani, B., "Extracting the Liver and Tumor from Abdominal CT Images", IEEE, Fifth International Conference on Signal and Image Processing (ICSIP)", pp. 122-125, 2014.

[4] Narinder Singh and SB Singh, "A Modified Mean Gray Wolf Optimization Approach for Benchmark and Biomedical Problems", Evolutionary Bioinformatics, Volume 13, pp 1-28, 2017.

[5] Russo F. "A method for estimation and filtering of Gaussian noise in images". IEEE Transactions on Instrumentation and Measurement. 52(4): 1148-54, Aug 2003.

[6] Sonali Patil VR. "Udupi Preprocessing to be considered for MR and CT Images Containing Tumors". IOSR Journal of Electrical and Electronics Engineering. Vol 1(4): 54-7, 2012.

[7] C Chaux L, Duval A, Benazza-Benyahia and Pesquet JC. "A nonlinear Stein-based estimator for multichannel image Denoising”. IEEE Transactions on Signal Processing, 56(8): 3855-70, 2008.

[8] Narain Ponraj D, Evangelin Jenifer M, Poongodi P, Samuel Manoharan J. "A Survey on the Preprocessing Techniques of Mammogram for the Detection of Breast Cancer". Journal of Emerging Trends in Computing and Information Sciences. December; 2(12); 2011.

[9] S. Gunasundari and M. Suganya Ananthi, "Comparison and Evaluation of Methods for Liver Tumor Classification from CT Dataset”, International Journal of Computer Applications, Volume 39- No. 18, 2012.

[10] K. Mala, V. Sadasivam, and S. Alagappan, "Neural Network based Texture Analysis of Liver Tumor from Computed Tomography Images", International Journal of Biological, Biomedical and Medical Sciences, Vol. 2 Issue 1, pp 33-37, 2007.

[11] Jayanthi, M., Kanmani, B., "Extracting the Liver and Tumor from Abdominal CT Images", IEEE, Fifth International Conference on Signal and Image Processing (ICSIP), pp. 122-125, 2014.

[12] Kumar SS, Moni RS, Rajeesh, I. "Automatic liver and lesion segmentation: a primary step in diagnosis of liver diseases Signal, Image and Video Processing”. 2011 March 31.

[13] Ahmed Fouad Ali, Abdalla Mostafa, Gehad Ismail Sayed, Mohamed Abd Elfattah, Aboul Ella Hassanien, "Nature Inspired Optimization Algorithms for CT Liver segmentation”, 2016.

[14] Gehed Ismail Sayed, Aboul Ella Hassan and Gerald Scahefer, "An automated computer aided diagnosis system for abdominal CT liver images", International conference on medical imaging, understanding and analysis (Elseveir), pp 68-73, 2016.

[15] Mittal N, Singh U, Singh Sohi B. "Modified grey optimizer for global engineering optimization". Appl Comput Intel Soft Comput, 1-16, 2016.

[16] Edy Fradinata, Sakesun Suthumanon, Suntiamorntut, "Initial optimal parameters of artificial neural network and SVR”, International Journal of Electrical and Computer Engineering (IJECE), Volume 8, Issue No 5, 2018. http://doi.org/10.11591/ijece.v8i5.pp\%25p

[17] Ratna Nitin Patil, Dr Sharvavi Chandrashekhar Tamane, "A comparative analysis on the evaluation of classification algorithm in the prediction of diabetes", International Journal of Electrical and Computer Engineering (IJECE), Vol 8 , Issue 5, 2018. http://doi.org/10.11591/ijece.v8i6.pp\%25p

[18] Jayanthi, M., Kanmani, B., "Extracting Liver and Tumor from Computer Tomography Images Using Hybrid Techniques", International Journal of Innovations \& Advancement in Computer Science, Volume: 4(3): 12-17; 2015.

[19] Tomasi C, Manduchi R, "Bilateral Filtering Tomasi C, Manduchi R. 1998, Bilateral Filtering for Gray and Images". India, Bombay: Proceedings of the IEEE International Conference on Computer Vision. p. 834-46, 1998.

[20] Kumar SS, Moni RS, Rajeesh, I. "Automatic liver and lesion segmentation: a primary step in diagnosis of liver diseases Signal, Image and Video Processing”. March 31, 2011.

[21] S. Mirjalili, S. Saremi, S.M. Mirjalili, and L.D.S. Coelho, "Multi-objective grey wolf optimizer: A novel algorithm for multicriterion optimization", Expert Syst. Appl., vol. 47, pp. 106-119, Apr 2016.

[22] Mirjalili, and A. Lewis, "Grey wolf optimizer", Adv.Eng. Softw. vol. 69, pp. 46-61, Mar. 2014.

[23] M. Jayanthi, "Segmentation of Liver Abnormality based on Label Connected Component Algorithm", Inter. Journal of Scientific Engineering and Technology. Volume: 6, Issue: 7, PP: 247-249; 2017.

[24] The cancer Imaging Archive (TCIA), http://www.cancerimagingarchive.net/

[25] M. Jayanthi, "Comparative Study of Different Techniques Used for medical image segmentation of Liver from Abdominal CT Scan", IEEE WiSPNET 2016 conference; pp. 1462-1465, 2016. 


\section{BIOGRAPHIES OF AUTHORS}
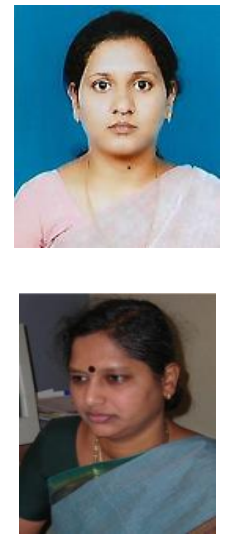

M. Jayanthi is Senior Assistant Professor in Department of Electronics and Communication Engineering, New Horizon College Of Engineering, Bengaluru and Karnataka, India. She received her M.E degree in Communication Systems from Mepco Schlenk Engineering college, Sivakasi, Tamilnadu in the year 2006.She is pursuing the Ph.D degree in the Department of ECE, BMS college of Engineering, Bengaluru. Her research interests include image processing, Digital Signal processing and Biomedical signal processing. She has 11 years of experience in teaching. Email:jayanthi.sathishkumar@live.com

B Kanmani, Ph.D is Professor and Dean Academics, Department of Telecommunication Engineering, BMS college of Engineering, Bengaluru. She received her Bachelors in Electronics and Communication Engineering from Nagarujuna University in 1987, M.Tech. degree in Digital communication from Indian Institute of Technology, Kanpur in 1990, and PhD from the Indian Institute of Science Bangalore (IISc) in the year 2006. She has been with BMS College of Engineering, Bangalore, since 1995, and has to her credit 25 International publications, of which three are in Journals. She is a supervisor for the Ph.D student at Visvesvaraya Technological university, Belagavi. She teaches under-graduate courses on Analog Signal Processing, Digital Signal Processing, Analog Communication and Digital Communication.She is Senior Member IEEE, Member Tata Library and Life Member ISTE. 